New study suggests na notubes have a use in the fight aga inst $E$. coli, and could possibly be used in the production of antimic robial materials.

\title{
Fighting bacteria using nanotubes
}

A recent study provides evidence that single-walled carbon nanotubes

(SW C N Ts) can kill bacteria, such as Escherichia coli (a common pathogen), by severely damaging their cell walls.

"We began the study out of concerns for the possible toxicity of nanotubes in aquatic environments and their presence in the food chain," said M enachem Elimelech, senior author on the paper. "W hile nanotubes have great promise for medical and commercial applications, there is little understanding of how they interact with humans and the environment."

'This study has been desc ribed as the first direct evidence of the strong a ntimic robial a c tivity of carbon na notubules.

This discovery could help in the fig ht a ga inst a ntibiotic -resista nt infections.'

"T he nanotubes are microscopic carbon cylinders, thousands of times smaller than a human hair that can be easily taken up by human cells," said Elimelech. "We wanted to find out more about where and how they are toxic."

This study, from Yale U niversity (CT, USA) and published in Small, has been described as the first direct evidence of the strong antimicrobial activity of CN Ts. This discovery could hel $p$ in the fight against antibiotic-resistant infections.

E. coli was incubated with SW CN Ts for up to $1 \mathrm{~h}$. The authors used a pristine SW CNT with a narrow diameter distribution to demonstrate that direct contact of SW CNT aggregates with $E$. coli resulted in bacterial membrane damage, which is the probable mechanism that leads to subsequent bacterial cell death. The authors have speculated that the SW CNTS, which are long and thin, puncture the cells and cause the cellular damage.
The authors have also discounted metal toxicity as a mechanism for the cell damage. The SW CN Tswere synthesized and purified rigorously to avoid the metal contaminants of commercial sources. Their toxicity could bemanaged in future research and for human use by embedding them to prevent their leaching into the environment.

"We're now studying the toxicity of multiwalled carbon nanotubes and our preliminary results show that they are less toxic than SW C N Ts," Elimelech said. "We are also looking at the effects of SWCN Ts on a wide range of bacterial strains to better understand the mechanism of cellular damage."

From these results, the authors believe that SW CN Ts could be used to create antimicrobial materials and surface coatings to improve hygiene.

Source: Kang S, Pinault M , Pfefferle LD, Elimelech M : Single-walled carbon nanotubes exhibit strong antimicrobial activity. Langmuir 23(17), 8670-8673 (2007). 


\section{Wearing a coat enables nanowires to enter cells easily}

'Nanowires, as well as other

na nomaterials, have great

potential as new drug-delivery

systems because they

can penetrate tumors more easily.'

Researchers have developed a protein coating that might turn nanowires into a new drug-delivery system that could enable the use of lower doses of medicines that are harmful to normal cells.

Silica nanowires introduced the Shiga toxin type 1 A subunit (StxA1) into cultured bovine and human epithelial cells. Technology used in the laboratory previously enabled researchers to coat the nanowires with StxA1 and fibronectin, which induces the integrin-mediated uptake of nanowires. Complex interactions among fibronectin, StxA1 and the nanowires were detected.

Internalization of the nanowire-StxA1-fibronectin complexes and their ability to cause cell death were measured by neutral red cytotoxicity assays and field emission scanning electron microscopy.

Nanowires, as well as other nanomaterials, have great potential as new drug-delivery systems because they can penetrate tumors more easily and can be coated with factors that can target cells/tumors while sparing normal cells. $\mathrm{H}$ owever, determining methods by which nanowires and other nanomaterials can penetratecells easily is a difficulty that must be overcome.
'Further studies a re in progress to improve the func tiona lization of the nanowires.'

The research from the U niversity of Idaho (USA) and Seoul N ational University (South Korea) indicates that nanowires can carry StxA1 and, potentially, other toxic or therapeutic agents, into eukaryotic cells. Further studies are in progress to improve the functionalization of the nanowires in the hope of increasing the efficiency of internalization and also to substitute ligands for specific celltargeting factors.

Source: Kwon N H, Beaux II M F, Ebert C et al.: $\mathrm{N}$ anowire-based delivery of Escherichia coli 0157 Shiga toxin $1 \mathrm{~A}$ subunit into human and bovine cells. N ano Lett. 7(9), 2718-2723 (2007).

\section{N ew website offers nano-advice on hazards and risks}

'The site provides impartial advice to those in industry, academia and the widerpublic concerning the potential risks

to human health and the environment from nanoma teria ls.'

SAFEN AN 0 is a new website providing information relating to nanotechnology health and safety. It is being managed by the Institute of $O$ ccupation $M$ edicine ( $\mathrm{OM}$ ) (Edinburgh, UK) and aims to become the UK's premier resource on nanotechnology hazard and risk. The site provides impartial advice to those in industry, academia and the wider public concerning the potential risks to human health and the environment from nanomaterials.
Director of theSAFEN AN 0 initiative is Rob Aitken, who is D irector of Strategic Consulting, having been Director of Research D evelopment at the $I O M$. The IOM is one of the UK's foremost promoters of responsible nanotechnology development and possesses extensive experience concerning the potential risks from particles and fibers. A combination of this expertise and state-of-art techniques al lows the IO M to extend current knowledge and facilitate comparisons between existing substances and new nanoparticles.

SAFEN AN 0 has access to a multidisciplinary consortium of internationally recognized researchers through the Safety of $\mathrm{N}$ anoparticles Interdisciplinary R esearch C entre (SnIRC). SnIRC was formed by the IO M in 2004 to address nanoparticle
'SAFENANO has access to a multid isc ip lina ry consortium of intemationa lly recognized researchers through the Safety of Nanopartic les Interd isc ip lina ry Research Centre.'

risk issues, and is based on long-standing collaborations between the $\mathrm{IO} \mathrm{M}$ and Edinburgh, Aberdeen and $\mathrm{N}$ apier Universities.

The initiative and site has been designed to help industrial and academic communities to quantify and control the risks to their workforce, as well as to consumers, the general population and the environment, through both information provision and consultancy services. Source: SAFEN AN O website (www.safenano.org). 


\section{in brief...}

Twenty-eight-day inhalation toxicity study of silver nanoparticles in Sprague-Daw ley rats. Ji JH, Jung JH, Kim SS et al.: Inhal. Toxicol. 19(10), 857-871 (2007).

Silver nanoparticles (Ag NPs), owing to their antibacterial properties, have been used in health, electronic and home products. Thus, the potential inhalation toxicity of Ag NPs is of increasing importance to health professionals and consumers. These authors used a device that generates Ag NPs by evaporation/condensation using a small ceramic heater to evaluate the effect of the inhalation of nanosized particles on the respiratory system. The device distributed the desired concentrations of Ag NPs to chambers containing experimental animals. The concentrations and distribution of the Ag NPs with respect to size were also measured directly.

In vivo imaging of hydrogen peroxide with chemiluminescent nanoparticles.

Lee D, Khaja S, Velasquez-Castano JC et al.: Nature M ater. DOI: 10.1038/nmat1983 (2007) (Epub ahead of print).

This study shows that nanoparticles formulated from peroxalate esters and fluorescent dyes can image hydrogen peroxide, overproduction of which is implicated in disease development, in vivo with high specificity and sensitivity. The peroxalate nanoparticles undergo a three-component chemiluminescent reaction between hydrogen peroxide, peroxalate esters and fluorescent dyes and have several attractive properties for in vivo imaging. The authors believe that they will have numerous applications in the in vivo imaging of hydrogen peroxide because of their high specificity/sensitivity and deep tissue imaging capabilities shown in this research.

Tw o-photon excitation fluorescence cross-correlation assay for ligand-receptor binding: cell membrane nanopatches containing the human $\boldsymbol{\mu}$-opioid receptor. Swift JL, Burger MC, Massotte D, Dahms TE, Cramb DT: Anal. Chem. 79(17), 6783-6791 (2007). The authors describe the introduction of two-photon excitation fluorescence crosscorrelation spectroscopy (TPE-FCCS) to the direct analysis of ligand-receptor interactions of the human $\mu$-opioid receptor (hM OR) for both agonists and antagonists. Fluorescently distinct, dye-labeled hM OR-containing cell-membrane nanopatches ( $100-\mathrm{nm}$ radius) and ligands were developed to enable measurement of the dissociation constant $(\mathrm{Kd})$ of the ligand-receptor binding for G-proteincoupled receptors. The assay, when ligands are QD labeled, can detect binding with ligand concentrations in the subnanomolar regime.

\section{Clinical trial results of nanoemulsion for serious skin infections}

A study of patients with onychomycosis (toenail fungus) has shown that a new topical lotion can penetrate the skin deeply enough to target and eliminate serious skin infections without being systemically absorbed and has also shown a high degree of safety and tolerability.

The 47th Annual Interscience C onference on Antimicrobial Agents and Chemotherapy (ICAAC) meeting in September saw the Phase I data for the new lotion, N B-002, presented. N B-002 is a topical oil-in-water nanoemulsion combined with an antimicrobial agent that is used commonly in oral products for treating gingivitis and other mouth and throat conditions.

"At present, the vast majority of people with onychomycosis are untreated as a result of concerns for the serious toxicities associated with the available oral medications," said James Baker, chief science officer and founder of $\mathrm{N}$ anoBio C orporation in Ann Arbor, M I, U SA. "O ur safety data, combined with early information from the ongoing Phase II trial, indicate that N B-002 is potentially the first treatment for onychomycosis that is highly efficacious and very safe."

To 'nanosize' the particles, making them small enough to enter the skin through pores and hair follicles but too large to penetrate the epithelium tight junctions, the N B-002 nanoemulsion undergoes a high-energy process. Therefore, N B-002 is not absorbed systemically.

A total of 20 patients with advanced study to receive two strengths of N B002. Affected and unaffected toenails, as well as a small surrounding area of skin, where treated twice daily for $\mathbf{2 8}$ days. Blood samples were taken periodically to determine whether the drug was absorbed systemically.

Throughout the study period, the drug was below the quantifiable limit of systemic absorption for all subjects and, unlike current onychomycosis treatments, N B-002 had no safety or dermal irritation concerns. A Phase II clinical trial for onychomycosis is ongoing with results expected in early 2008.

"Results of the Phase 1 clinical trial are important to the fields of dermatology and infectious diseases because currently approved systemic medications for onychomycosis carry serious risks of cardiac and liver toxicity," said James Baker, whose team developed the lotion.

Source: 47th Annual Interscience Conference on Antimicrobial Agents and Chemotherapy

(ICAAC) meeting (www.icaac.org/) onychomycosis were randomized in the

\section{About the Bulletin Board}

The Bulletin Board highlights some of the most important events and launches in nanomedicine and nanotechnology research. The editorial team welcomes suggestions for timely, relevant items. If you have newsworthy information, please contact:

Morag Robertson, PhD,

Editor, Nanomedicine,

Future Medicine Ltd, Unitec House,

2 Albert Place, London N3 1QB, UK

E-mail: m.robertson@futuremedicine.com

Tel: $+44(0) 2083716090$;

Fax: $+44(0) 2083432313$ 